sulfiram-like reaction with metronidazole and ethanol. Ann Pharmacother 2002; 36: 971-974.

5. Stockley's Drug Interactions. Alcohol + metronidazole and related drugs. 2016. Available at: https://www. medicinescomplete.com/\#/content/stockley/x020033\#00000166 (accessed April 2020).

6. Mergenhagen K A, Wattengel B A, Skelly M K et al. Fact vs. fiction: the evidence behind alcohol and antibiotic interactions, a review. Antimicrob Agents Chemother 2020; 64: pii: e02167-19.

https://doi.org/10.1038/s41415-020-2012-x

\section{Oral surgery}

\section{New MRONJ guidance}

Sir, there has been guidance for dentists on the prevention of medication-related osteonecrosis of the jaws (MRONJ) for several years. ${ }^{1}$ Although this guidance also included information for prescribers and dispensers, it does not appear to have been widely disseminated. Indeed, only $4 \%$ of general medical practitioners in a Birmingham study were aware of MRONJ guidelines. ${ }^{2}$ I therefore welcome the publication by the Royal College of Physicians for the multi-disciplinary team. ${ }^{3}$

MRONJ is defined as exposed bone, or bone that can be probed through a fistula, in the maxilla or mandible, that has been present for more than eight weeks. ${ }^{4}$ It is an adverse side effect of anti-angiogenic or anti-resorptive medication. ${ }^{1,3}$ The incidence of MRONJ in patients who take these medications is reported as $1 \%$ in cancer patients and $0.1 \%$ in patients with metabolic bone disease. ${ }^{1}$

There have been instances of MRONJ occurring spontaneously, but it is most common following a dental extraction. ${ }^{1,3,4}$ Prevention of MRONJ involves pretreatment screening, extraction of teeth with poor prognosis, adjustment of prostheses and education, including oral hygiene instruction and controlling risk factors such as smoking and alcohol. This has been shown to reduce the incidence of MRONJ by up to $50 \%{ }^{5}$

Dentists have reported poor communication with other healthcare professionals and often rely on patient recall for their medical history. ${ }^{6}$ The new guidance introduces dental alert cards which are to be carried by patients and shown to their dentists. ${ }^{3}$ Referral and reply letters between oncology, dentists and maxillofacial/oral surgeons have also been produced.

We should welcome and familiarise ourselves with these new methods of communication and remember to report all cases of suspected MRONJ to the MHRA.? Reducing the incidence of MRONJ will have financial benefit for the NHS, as this is a difficult condition to manage, and for severe cases may require surgery for debridement or resection. ${ }^{4}$

\section{Frazer-Cox, North Wales, UK}

\section{References}

1. Scottish Dental Clinical Effectiveness Programme. Oral health management of patients at risk of medication-related osteonecrosis of the jaws. 2017. Available at: http:// www.sdcep.org.uk/published-guidance/medication-related-osteonecrosis-of-the-jaw/ (accessed July 2020).

2. Rahman Z, Nayani S, Anstey H, Murphy M J. A survey evaluating the awareness of MRONJ within the Birmingham GMP community. Oral Surg 2018. Available at: https://doi.org/10.1111/ors.12372 (accessed July 2020).

3. Royal College of Physicians. Medication-related osteonecrosis of the jaw: guidance for the oncology multidisciplinary team. 2019. Available at: https:// www.rcplondon.ac.uk/guidelines-policy/medication-related-osteonecrosis-jaw-guidance-oncology-multidisciplinary-team (accessed July 2020).

4. Ruggiero S L, Dodson T B, Fantasia J et al. American Association of Oral and Maxillofacial Surgeons position paper on medication-related osteonecrosis of the jaw - 2014 update. J Oral Maxillofac Surg 2014; 72: 1938-1956.

5. Vandone A M, Donadio M, Mozzati M et al. Impact of dental care in the prevention of bisphosphonate-associated osteonecrosis of the jaw: a single-center clinical experience. Ann Oncol 2012; 23: 193-200.

6. Sturrock A, Preshaw P M, Hayes C, Wilkes S. General dental practitioners' perceptions of, and attitudes towards, improving patient safety through a multidisciplinary approach to the prevention of medication- related osteonecrosis of the jaw (MRONJ): a qualitative study in the North East of England. BMJ Open 2019; 9: e029951. doi:10.1136/bmjopen-2019-029951.

7. Yellow Card. Report online: www.mhra.gov.uk/ yellowcard; via the Yellow Card App or by emailing yellowcard@mhra.gov.uk.

https://doi.org/10.1038/s41415-020-2013-9

\section{Dental education}

\section{Undergraduate research}

Sir, I read with interest a colleague's letter on the subject of getting published. ${ }^{1} \mathrm{I}$ believe that research experience is an area in which many dentists including dental core trainees (DCTs) do not have any substantial experience. Although the importance of research is touched upon at undergraduate level, there are few opportunities and most of the research activity in dental schools is carried out by postgraduate students. Many dental students would benefit from a further insight into research or opportunity to carry out small research projects at an undergraduate level supervised by senior staff. This would also give them a good foundation for future projects at a postgraduate level.

At a DCT level academic clinical fellow posts address this ability for trainees to be able to carry out research; however, these are few and far between. Some DCT posts may provide an insight into research such as critical appraisal, however, I feel there should be more scope to incorporate further research opportunities into these posts. Many trainees would be willing to assist senior colleagues in established projects or like the chance to complete small projects of their own with guidance and assistance to provide good learning opportunities as well as supporting portfolios.

G. K. Randhawa, Bristol, UK

\section{Reference}

1. Jackson M. How to get published? Br Dent J 2020; 228: 317-318.

https://doi.org/10.1038/s41415-020-2014-8

\section{Periodontology}

\section{Periodontal therapy and cell adhesion}

Sir, periodontitis is a chronic infection and inflammation of the periodontium. ${ }^{1} \mathrm{~A}$ relationship between periodontal therapy (PT) and reduction in risk of cardiovascular diseases has been reported. ${ }^{2}$ One hypothesis is that PT reduces the expression of cell adhesion molecules that are pivotal in the pathogenesis of vascular inflammation and atherosclerosis. ${ }^{3}$ This first meta-analysis included eight studies (three randomised control trials, one prospective nonrandomised study, and four retrospective). The main outcome was change in the levels of cell adhesion molecules (soluble endothelial selectin s-E-selectin-1, soluble intercellular adhesion molecule-1 s-ICAM-1, and soluble vascular cell adhesion molecule-1 s-VCAM-1) following PT. Standardised difference in means (SDM) was used for effect size (ES) measurement. An ES of 0.20.5 is considered small, $0.5-0.8$ is medium, and more than 0.8 is large.

Of the 797 patients ( 445 in PT, 352 in control), men formed $62.3 \%$ (497) with $69.8 \%$ (311) and $52.8 \%$ (186) in the respective groups; the mean age of patients was 51.9 and 55.89 years respectively. PT included oral hygiene instructions, scaling and root planing, antibiotics, chlorhexidine mouthwashes, tooth extractions and surgical PT. The control group received no treatment. Patients had moderate to severe periodontitis. Follow up varied between 3-6 months. PT showed a positive and medium effect size (SDM 0.52, 95\% CI 0.10-0.94) on decreasing s-E-selectin values at follow up. PT did not show a statistically significant effect size on lowering s-ICAM-1 (SDM 0.41, 